Journal of Patient-Centered

$1-27-2020$

\title{
In Gratitude of 2019 JPCRR Peer Reviewers
}

Follow this and additional works at: https://aah.org/jpcrr

Part of the Medical Humanities Commons, and the Other Medicine and Health Sciences Commons

\section{Recommended Citation}

In gratitude of 2019 JPCRR peer reviewers. J Patient Cent Res Rev. 2020;7:63. doi: 10.17294/

2330-0698.1748

Published quarterly by Midwest-based health system Advocate Aurora Health and indexed in PubMed Central, the Journal of Patient-Centered Research and Reviews (JPCRR) is an open access, peer-reviewed medical journal focused on disseminating scholarly works devoted to improving patient-centered care practices, health outcomes, and the patient experience. 


\section{In Gratitude of 2019 JPCRR Peer Reviewers}

The editors of Journal of Patient-Centered Research and Reviews sincerely appreciate the reviewers of manuscript submissions from the past calendar year. Quality assurance of the content published within the journal is largely dependent on the volunteer contributions of these individual experts.

Rasha Alkhatib

Andy Anderson

Stephen Asche

Deanna Attai

Diane Austin

Dennis Baumgardner

Jean Baumgardner

Lisa Berger

John Brill

Roger Brown

Brian Buggy

Alyson Capp

Laura Colicchia

Thomas Cornwell

Jennifer Deal

Malini DeSilva

Thomas Dilworth

Lee Dresang

Alison Essary

Nicole Eull

Adam Farmer

Mustafa Farooque

Brenda Fay

Douglas Fernald

Veronica Fitzpatrick
Sarah Greene

Joe Grundle

Chris Hallberg

David Hamel

Kayla Heslin

Clifford Hudis

M. Fuad Jan

Anne Jones

Ariba Khan

Christopher Klink

Darly Knoedler

Fabiana Kotovicz

Jessica Kram

Sharon Laing

Yangyang Liu

Brittany Long

Kate Louther

William MacDonald

Robert Marrs

Jeff Melbye

Joshua Mersky

Alyssa Mohorek

Chris Morley

Ana Perez Moreno

Bruce Morgenstern
Alicen Nelson

Frank Rhame

Mark Robinson

Steve Rommelfanger

Richard Rovin

MaryAnne Scherer

Sarina Schrager

Rebecca Schultz

Joel Shoolin

Matthew Simpson

Michelle Simpson

Sally Smaida

Stephen Sokalski

Leif Solberg

Natalie Sorenson

Lisa Sullivan Vedder

Glenda Sundberg

Shanita Thomas

Michael Thompson

Judy Tjoe

Mindy Waite

Jasmine Wiley

Jun Yin

Staci Young

Jodi Zilinski

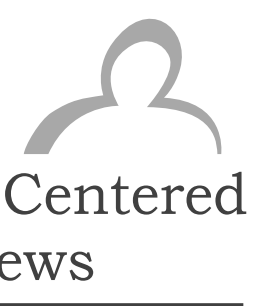

